\title{
Corrigendum
}

\section{Market equilibrium with heterogeneous recursive-utility-maximizing agents ${ }^{\star}$}

\author{
Chenghu Ma \\ Department of Economics, McGill University, Montreal, P.Q., CANADA H3A 2T7
}

August 23, 1995

Summary. The paper by C. Ma [1] contains several errors. First, statement and proof of Theorem 2.1 on the existence of intertemporal recursive utility function as a unique solution to the Koopmans equation must be amended. Several additional technical conditions concerning the consumption domain, measurability of certainty equivalent and utility process need to be assumed for the validity of the theorem. Second, the assumptions for Theorem 3.1 need to be amended to include the Feller's condition that, for any bounded continuous function $f \in C\left(S \times \mathscr{R}_{+}^{n}\right)$, $\mu\left(f\left(\tilde{s}_{t+1}, \theta\right) \mid s_{t}=s\right)$ is bounded and continuous in $(s, \theta)$. In addition, for Theorem 3.1, the price $p$, the endowment $e$ and the dividend rate $\delta$ as functions of the state variable $s \in S$ are assumed to be continuous.

The Feller's condition for Theorem 3.1 is to ensure the value function to be well-defined. This condition needs to be assumed even for the expected additive utility functions (See Lucas [2]). It is noticed that, under this condition, the right hand side of equation (3.5) in [1] defines a bounded continuous function in $s$ and $\phi$. The proof of Theorem 3.1 remains valid with this remark in place.

A correct version of Theorem 2.1 in [1] is stated and proved in this corrigendum. Ozaki and Streufert [3] is the first to cast doubt on the validity of this theorem. They point out correctly that additional conditions to ensure the measurability of the utility process need to be assumed. This condition is identified as condition $C E_{4}$ below. In addition, I notice that, the consumption space is not suitably defined in [1], especially when a unbounded consumption set is assumed. In contrast to what claimed in [3], I show that the uniformly bounded consumption set $X$ and stationary information structure are not necessary for the validity of Theorem 2.1 .

\section{An existence theorem of recursive utility}

Let $X=\mathscr{R}_{+}^{l}$ be the current consumption set. Let $(\Omega, \mathscr{F}, P)$ be a probability space, and let $\mathscr{F}_{0}=\{\varnothing, \Omega\},\left\{\mathscr{F}_{t}: t \geq 0\right\}$ be an increasing filtration of sub- $\sigma$-algebras of

\footnotetext{
* I would like to thank Hiroyuki Ozaki and Peter Streufert for pointing out correctly some mistakes made in the original article. Comments and suggestions from an anonymous referee are gratefully appreciated. Financially support from SSHRC of Canada is acknowledged.
} 
$\mathscr{F}$ that represents the information structure. The space of consumption programs, denoted by $D_{+}$, is defined as the positive cone of Banach space $\left(D,\|\cdot\|_{D}\right)$ :

$\left\{d=\left(c_{0}, \ldots, c_{t}, \ldots\right): c_{t} \in \mathscr{R}^{l}\right.$ is $\left\{\mathscr{F}_{s}\right\}_{0 \leq s \leq t}$-measurable, and $\left.\|d\|_{D} \equiv \sup _{t, \omega} \lambda^{t}\left\|c_{t}(\omega)\right\|<\infty\right\}$, where $\|\cdot\|$ is the Euclidean norm on $\mathscr{R}^{l}$, and where $0<\lambda \leq 1$ is a constant that restricts the consumption growth rate. For any given consumption stream $d \in D_{+}$, a recursive utility process $U(d) \equiv\left\{U_{t}(d)\right\}_{t \geq 0}$ is defined recursively by the following Koopmans equations:

$$
U_{t}(d)=W\left(c_{t}, \mu\left(U_{t+1}(d)\right)\right), \quad t \geq 0 .
$$

\section{Assumptions for the aggregator $W(\cdot, \cdot)$}

$W_{1} W(\cdot, \cdot): X \times \mathscr{R}_{+}$is continuously differentiable, strictly increasing in both arguments, and $W(0,0)=0$

$W_{2} W(\cdot, \cdot)$ is jointly concave in both arguments

$W_{3} \exists \rho \geq 0$, s.t. $\sup _{x \in \mathscr{R}_{+}} \frac{W(x \vec{i}, 0)}{x^{\rho}} \equiv b<\infty$, where $\vec{i}=[1, \ldots, 1] \in \mathscr{R}^{l}$.

$W_{4} \exists \beta_{v} \in\left(0, \lambda^{\rho}\right)$, s.t. $W_{v}(c, v) \leq \beta_{v}, \forall(c, v) \in X \times \mathscr{R}_{+}$.

\section{Assumptions for the certainty equivalent $\mu(\cdot)$}

$C E_{1} \mu$ is a real function defined over $\mathscr{R}_{+}$-valued r.v.'s, that satisfies i) $\mu(\tilde{x})=x$, if $\tilde{x}=x$, a.e.; and ii) $\mu(\tilde{x}) \geq \mu(\tilde{y})$, if $\tilde{x} \geq \tilde{y}$, a.e., and the inequality is strict if $\tilde{x}>\tilde{y}$ with positive probability.

$C E_{2} \exists \beta_{\mu}>0, \beta_{\mu} \beta_{v}<\lambda^{\rho}$, s.t. $|\mu(\tilde{x})-\mu(\tilde{y})| \leq \beta_{\mu} \mu(|\tilde{x}-\tilde{y}|), \forall \tilde{x}, \tilde{y} \in \mathscr{R}_{+}$.

$C E_{3}$ For each $\tilde{x}$ and $\tilde{h}, \mu(\tilde{x}+\alpha \tilde{h})$ is concave in $\alpha$ on $\left\{\alpha \in \mathscr{R}: \tilde{x}+\alpha \tilde{h} \in \mathscr{R}_{+}\right.$, a.e. $\}$.

$C E_{4}$ For $\left\{\mathscr{F}_{s}\right\}_{0 \leq s \leq t+1}$-measurable random variable $x_{t+1}, \mu\left(x_{t+1} \mid \mathscr{F}_{t}\right)$ is $\left\{\mathscr{F}_{s}\right\}_{0 \leq s \leq t}$ measurable.

Under these assumptions on the recursive utility generator $(W, \mu)$, we can prove the existence of utility process as unique solution to the Koopmans equation:

Theorem 2.1. Under assumptions $W_{1}, W_{3}$ and $W_{4}$, and $C E_{1}, C E_{2}$ and $C E_{4}$, for any consumption program $d \in D_{+}$, the Koopmans equation generates a unique non-negative utility process $U(d)=\left\{U_{t}(d)\right\}_{t \geq 0} \in\left(\mathscr{U}_{+},\|\cdot\|_{\mathscr{U}}\right)$, where $\left(\mathscr{U},\|\cdot\|_{\mathscr{U}}\right)$ is defined by $\left\{\left(U_{0}, \ldots, U_{t}, \ldots\right): U_{t} \in \mathscr{R}\right.$ is $\left\{\mathscr{F}_{s}\right\}_{0 \leq s \leq t}$-measurable, and $\left.\|U\|_{\mathscr{O}} \equiv \sup _{t, \omega} \lambda^{\rho t}\left|U_{t}(\omega)\right|<\infty\right\}$.

Furthermore, if $W_{2}$ and $C E_{3}$ are also satisfied, the utility process will be concave in the sense that $U_{t}\left(\alpha d+(1-\alpha) d^{\prime}\right) \geq \alpha U_{t}(d)+(1-\alpha) U_{t}\left(d^{\prime}\right)$, for all $\alpha \in[0,1], t \geq 0$ and $d, d^{\prime} \in D_{+}$.

Proof: For any given $d \in D_{+}$, define operator $T_{d}: \mathscr{U}_{+} \rightarrow \mathscr{U}_{+}$by

$$
T_{d}(U)_{t}=W\left(c_{t}, \mu\left(U_{t+1} \mid \mathscr{F}_{t}\right)\right), \quad t \geq 0, U \in \mathscr{U} .
$$

Operator $T_{d}$ is well-defined: First, the right hand side is non-negative and $\mathscr{F}_{\mathrm{s}}$-measurable for all $0 \leq s \leq t$ by assumption $C E_{4}$ and $W_{1}$. Second, we can show 
that $\left\|T_{d}(U)\right\|_{\mathscr{U}}<\infty$ for all $U \in \mathscr{U}_{+}$. This is because, for all $t \geq 0$,

$$
\begin{aligned}
0 & \leq \lambda^{\rho t} T_{d}(U)_{t} \\
& \leq \lambda^{\rho t} W\left(\|d\|_{D} \lambda^{-t},\|U\|_{\mathscr{U}^{\prime}} \lambda^{-\rho(t+1)}\right) \\
& \leq b\|d\|_{D}^{\rho}+\beta_{v} \lambda^{-\rho}\|U\|_{\mathscr{U}}<\infty,
\end{aligned}
$$

where in the third inequality we make use of $W(c, v) \leq W(c, 0)+\beta_{v} v$.

Next, we show that for each $d \in D_{+}, T_{d}$ admits unique fixed point $U \in \mathscr{U}_{+}$, which is the unique solution to the Koopmans equation. The existence of fixed points follows by construction. For any given $U^{0} \in \mathscr{U}_{+}$, define a sequence $\left\{U^{n}\right\}_{n \geq 0} \subset \mathscr{U}_{+}$by $U^{n}=T_{d}\left(U^{n-1}\right), n=1,2, \ldots$. This sequence is a Cauchy sequence in the Banach space $\left(\mathscr{U},\|\cdot\|_{\mathscr{U}}\right)$ since, for any positive integers $n>m$ and $t \geq 0$, we have

$$
\begin{aligned}
& \lambda^{\rho t}\left|U_{t}^{n}-U_{t}^{m}\right| \\
& \quad=\lambda^{\rho t}\left|W\left(c_{t}, \mu\left(U_{t+1}^{n-1} \mid \mathscr{F}_{t}\right)\right)-W\left(c_{t}, \mu\left(U_{t+1}^{m-1} \mid \mathscr{F}_{t}\right)\right)\right| \\
& \quad \leq\left(\beta_{v} \beta_{\mu} \lambda^{-\rho}\right)\left\|U^{n-1}-U^{m-1}\right\|_{\mathscr{O l}}, \forall t \geq 0,
\end{aligned}
$$

and repeat this process, we have

$$
\begin{aligned}
& \left\|U^{n}-U^{m}\right\|_{\mathscr{U}} \\
& \quad \leq\left(\beta_{v} \beta_{\mu} \lambda^{-\rho}\right)^{m}\left\|U^{n-m}-U^{0}\right\|_{\mathscr{H}} \\
& \quad \leq \frac{\left(\beta_{v} \beta_{\mu} \lambda^{-\rho}\right)^{m}}{1-\beta_{v} \beta_{\mu} \lambda^{-\rho}}\left\|U^{1}-U^{0}\right\|_{\mathscr{U}}
\end{aligned}
$$

Therefore, $\left\{U^{n}\right\}$ converges to a unique limit point $U \in \mathscr{U}_{+}$. In particular, setting $n \rightarrow \infty$, we have, for all $t$ and $m$,

$$
\lambda^{\rho t}\left|U_{t}-U_{t}^{m}\right| \leq \frac{\left(\beta_{v} \beta_{\mu} \lambda^{-\rho}\right)^{m}}{1-\beta_{v} \beta_{\mu} \lambda^{-\rho}}\left\|U^{1}-U^{0}\right\|_{\mathscr{Q}} .
$$

Moreover, for each $t \geq 0$,

$$
\begin{aligned}
\lambda^{\rho t}\left|T_{d}(U)_{t}-U_{t}\right| & \leq \lambda^{\rho t}\left(\left|T_{d} U_{t}-T_{d} U_{t}^{m}\right|+\left|U_{t}^{m+1}-U_{t}\right|\right) \\
& \leq \beta_{v} \beta_{\mu} \lambda^{-\rho}\left\|U-U^{m}\right\|_{\mathscr{U}}+\frac{\left(\beta_{v} \beta_{\mu} \lambda^{-\rho}\right)^{m+1}}{1-\beta_{v} \beta_{\mu} \lambda^{-\rho}}\left\|U^{1}-U^{0}\right\|_{\mathscr{\ell}},
\end{aligned}
$$

for all $m>0$. Let $m \rightarrow \infty$, we show that $T_{d} U_{t}=U_{t}$ for all $t$.

To show the fixed point to be unique, suppose by contrary that $T_{d}$ has another fixed point $U^{\prime} \in \mathscr{U}_{+}$, and $U^{\prime} \neq U$. We have, for each $t \geq 0, \lambda^{\rho t}\left|U_{t}^{\prime}-U_{t}\right| \leq$ $\beta_{v} \beta_{\mu} \lambda^{-\rho}\left\|U^{\prime}-U\right\|_{\mathscr{U}}$, or, $\left\|U^{\prime}-U\right\|_{\mathscr{U}} \leq \beta_{v} \beta_{\mu} \lambda^{-\rho}\left\|U^{\prime}-U\right\|_{\mathscr{U}}$ which is possible only when $U^{\prime}=U$, a contradiction. For each $d \in D_{+}$, let $U^{*}(d) \in \mathscr{U}_{+}$be the unique fixed point of $T_{d}$. The map $U^{*}: D_{+} \rightarrow \mathscr{U}_{+}$is well-defined, and gives the utility process on the consumption domain $D_{+}$. These end the proof of the first part of Theorem 2.1. The proof to the second part of the theorem is straightforward and standard, and is thus omitted. 


\section{References}

1. Ma, C.: Market equilibrium with heterogeneous recursive-utility-maximizing agents. Economic Theory 3, 243-266 (1993)

2. Lucas, R. E.: Asset prices in an exchange economy. Econometrica 46, 1426-1445 (1978)

3. Ozaki, H., Streufert, P.: On Chenghu Ma's article "Market equilibrium with heterogeneous recursiveutility-maximizing agents". Mimeo, 1994 